\title{
Erratum to: A Regenerative Approach with Dermal Micrografts in the Treatment of Chronic Ulcers
}

\author{
Francesco De Francesco $^{1}$ Antonio Graziano ${ }^{2,3}$ - Letizia Trovato ${ }^{2}$.

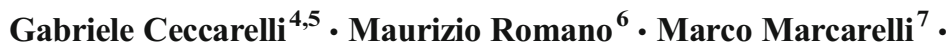 \\ Gabriella Maria Cusella De Angelis ${ }^{4,5}$ - Umberto Cillo ${ }^{6}$ - Michele Riccio ${ }^{8}$. \\ Giuseppe Andrea Ferraro ${ }^{1}$
}

Published online: 9 November 2016

(C) Springer Science+Business Media New York 2016

Erratum to: Stem Cell Rev and Rep

DOI 10.1007/s12015-016-9692-2

The original version of this article unfortunately contained a mistake. The name of the seventh author was incorrectly listed as Gabriella Maria Casella De Angelis, when it is actually Gabriella Maria Cusella De Angelis. The correct information is as shown above.

The original article was corrected.

The online version of the original article can be found at http://dx.doi.org/10.1007/s12015-016-9692-2

\section{Francesco De Francesco}

fran.defr@libero.it

1 Multidisciplinary Department of Medical-Surgical and Dental Specialties, Second University of Naples, Miraglia Square, 80122 Naples, Italy

2 Human Brain Wave srl, Corso Galileo Ferraris 63, 10128 Torino, Italy

3 SHRO - Temple University of Philadelphia, Philadelphia, PA, USA

4 Department of Public Health, Experimental Medicine and Forensics, University of Pavia, Pavia, Italy

5 C.I.T., Tissue Engineering Centre, University of Pavia, Pavia, Italy

6 Department of Surgery, Oncology and Gastroenterology, Hepatobiliary Surgery and Liver Transplantation, Padua University Hospital, Padua, Italy

7 Moncalieri Hospital, ASL To5 - U.C.S. Orthopedics and Traumatology, Piazza A. Ferdinando, 3, 10024 Moncalieri TO, Italy

8 Department of Reconstructive Plastic Surgery-Hand Surgery, AOU "Ospedali Riuniti", Ancona, Italy 